\title{
On Depression and Mental Disorder in South Africa
}

\section{Mncedisi Michael Willie*}

\author{
Multinum, Postnet Suite 427, Private Bag X37, Lynwood ridge, South Africa
}

${ }^{*}$ Corresponding author: Mncedisi Michael Willie, Multinum, Postnet Suite 427, Private Bag X37, Lynwood ridge, South Africa, Tel: +2776122 2369; E-mail: mwillie@multinum.co.za

Received date: December 04, 2017; Accepted date: December 08, 2017; Published date: December 15, 2017

Citation: Willie MM (2017) On Depression and Mental Disorder in South Africa. Med Clin Rev. Vol. 3 No. 4: 19.

Copyright: (c) 2017 Willie MM. This is an open-access article distributed under the terms of the Creative Commons Attribution License, which permits unrestricted use, distribution, and reproduction in any medium, provided the original author and source are credited.

\section{Editorial}

\section{Depression (Stress)}

Stress is how the body counters to certain endangerments, this may be either behavioural or a physiological in nature. In recent years all around the world, people are faced with challenges emanating from different types of settings consequently these may result in a stressful state of mind [1] Some of the causes of stress are as a result of external factors which may range from the political climate of the country to societal. Economic uncertainty undoubtedly impacts on high levels of stress. Contrariwise, human beings are competitive in nature, aspects such as a constant need to compete in challenging economic times, thriving for success is vital to them. Some of the other key aspects that contribute or result in stress include:

- Finding it very difficult to relax.

- Inability to say No to extra work or responsibility in the workplace or otherwise.

- Pressure such as getting to work on time.

- Pressure to meeting deadlines.

There is a body of literature that reveals stress as positively associated with suicide. According to van Heeringen $\mathrm{K}$ [2] there is correlation between increased levels of hopelessness and an increased risk of suicide in depressed individuals. This is further supported by Shahtahmasebi S [3] who examined the assertion that $80-90 \%$ of suicide cases had depression. Research conducted in KwaZulu Natal province in South Africa shows that more than ten percent of non-natural deaths are due to suicide [4].

Another study by Engelbrecht $\mathrm{C}$ et al. [5] revealed that suicide constitutes almost $10 \%$ of all fatalities admitted to the Pretoria Medico-Legal Laboratory, confirming suicide as one of the primary cause of mortality in South Africa.

\section{Mental Health}

Most common cause of deaths are not as a result of mental illness, however a greater proportion of those that die by suicide have some association with a mental diagnosis.
According to Burns JK [6], mental disorders are common and responsible for increased mortality due to suicide and reduced life expectancy. A longitudinal study by Teismann T, et al. [7] revealed that mental health moderates the association between depression and suicide ideation. Another study depicts that mental disorders (particularly depression and substance abuse) as often associated with cases of suicide [8].

Some commentators are of the view that critical aspects of mental health are not being prioritised and also not given the attention they require as a result affected individuals remain neglected. Ehiemua S [9] states that, lack of a consistent and coherent message about mental ill-health have ensured that it has remained untreated. There are also Studies that have shown that South Africans have a $30 \%$ chance of suffering from a mental disorder, and depression was found to be the most common mental disorder [10]. Williams $D$ et al. [11] depict psychiatric disorders as much higher in South African than other African countries such as Nigeria.

\section{Public sector}

In South Africa, Mental disorder does not seem to be a key priority, both from resource allocation and funding perspective, this is observed in both the private and public sector. It is estimated that $75 \%$ of people with a mental disorder in South Africa do not receive mental health services they need $[6,12]$. Furthermore, less than five percent of the health budget goes towards mental health. Inadequate resource planning which is also a function of under budgeting or lack of funding thereof for mental health patients has had a dire hard consequences in the public sector.

The Gauteng province in South Africa recently saw death of more than 100 mental health patients. These are patients who were transferred from Life Esidimeni - Life Healthcare (As a fully owned subsidiary of the Life Healthcare Group, we have been delivering healthcare and related services to the public sector (under contract to Department of Health) for over five decades) to ill-equipped non-government institutions. Thus implying possible inadequate resources planning in transferring these patients as well as possible administrative inefficiencies might have contributed to the deaths. This further depicting a dire need to treat mental health as a major 
health policy and social level in order to avoid such unfortunate events.

\section{Private sector}

Similarly, with the public sector, there are also challenges in the private sector where mental health disorders are not funded except a handful set of conditions. Medical scheme (health insurance carriers in the private sector) members encounter challenges when dealing with complex medical scheme rules. Mental illnesses are covered by prescribed minimum benefits (PMBs), the medical scheme is required by the Medical Schemes Act (Act 131 of 1998) to provide cover for the consultation(s) and other appropriate investigations that are necessary to diagnose a mental disorder Unfortunately, not all mental disorders are included in the PMB package. The following mental conditions are a handful set of conditions that qualify as PMBs as per the Medical Schemes Act (Act 131 of 1998):

- Acute stress disorder accompanied by recent significant trauma, including physical or sexual abuse.

- Major affective disorders, including unipolar and bipolar depression.

- Schizophrenic and paranoid delusional disorders.

\section{Conclusion}

Lack of adequate prioritisation, resource allocation and funding of mental disorder illness causes a serious concern for the livelihood of the affected lives. This is more prevalent in the death of more than 100 patients as a result of possible inadequacies in the public sector. Mental health and depression illnesses need to be promoted as a major health policy objective. Key proposals that could potentially work for both the public sector and private sector is integrating mental health treatment into primary health care. A policy shift that has been proposed would be to advocate for the inclusion of mental illness into Millennium Development Goals [13]. This will ensure that health ministries across the globe give this condition a priority it deserves, potentially influence the prioritisation in view of budget allocation and resource allocations.

\section{References}

1. Anisman H, Merali Z (1999) Understanding stress: characteristics and caveats. Alcohol Res Health 23: 241-249.
2. van Heeringen K (2012) Stress-diathesis model of suicidal behavior. In The Neurobiological Basis of Suicide (ed Dwivedi Y). CRC Press: 113-124.

3. Shahtahmasebi $S$ (2013) Examining the claim that $80-90 \%$ of suicide cases had depression. Frontiers in Public Health 1: 62.

4. Naidoo SS, Schlebusch L (2014) Sociodemographic characteristics of persons committing suicide in Durban, South Africa: 2006- 2007. Afr J Prim Health Care 6: 568-575.

5. Engelbrecht C, Blumenthal R, Morris NK, Saayman G (2017) Suicide in Pretoria: A retrospective review, 2007-2010. S Afr Med J 107: 715-718.

6. Burns JK (2014) The burden of mental disorders in KwaZuluNatal: mapping the treatment gap. South Afr J Psych 20: 6-10.

7. Teismanna T, Forkmannb T, Brailovskaiaa J, Siegmanna $P$, Glaesmerc H, et al. (2017) Positive mental health moderates the association between depression and suicide ideation: A longitudinal study. Int J Clin Health Psychol.

8. Pompili M, Serafini G, Innamorati M, Dominici G, Ferracuti S, et al. (2010) Suicidal Behavior and Alcohol Abuse. Int J Environ Res Public Health 7: 1392-1431.

9. Ehiemua S (2014) Mental Disorder: Mental health remains an invisible problem in Africa. European Journal of Research and Reflection in Educational Sciences 2: 11-16.

10. Jack H, Wagner RG, Petersen I, Thom R, Newton CR, et al. (2014) Closing the mental health treatment gap in South Africa: a review of costs and cost-effectiveness. Glob Health Action 7: 23431.

11. Williams DR, Herman A, Stein DJ, Heeringa SG, Jackson PB, et al. (2008) Twelve-month mental disorders in South Africa: Prevalence, service use and demographic correlates in the population-based South African Stress and Health Study. Psychol Med 38: 211-220.

12. Kleintjes S, Lund C, Swartz L (2012) South African mental health care service user views on priorities for supporting recovery: Implications for policy and service development. Disabil Rehabil 34: 2272-2280.

13. Sachs SE, Sachs JD (2007) Mental health in the millennium development goals: not ignored. PLoS Med Public Libr Sci 4: 56-57. 